\title{
CLIFFORD TRANSLATIONS OF SYMMETRIC SPACES
}

\author{
v. OZOLS
}

\begin{abstract}
A direct proof, not using the classification of symmetric spaces, is given for the following characterization of Clifford translations in a symmetric space $M$ : An isometry $g$ is a Clifford translation of $M$ if and only if the centralizer $Z(g)$ of $g$ in the isometry group of $M$ is transitive on $M$. The proof uses a geodesic characterization of Clifford translations, and the subgroups $T^{(h)}$ of J. de Siebenthal.
\end{abstract}

Introduction. A Clifford translation of a metric space is an isometry which moves each point the same distance as every other point. These isometries have been studied in various special cases by a number of people, including W. K. Clifford, G. Vincent, J. A. Wolf, H. Freudenthal, and J. Tits. The most complete results are due to J. A. Wolf, who essentially classified the finite groups of Clifford translations of compact simply connected symmetric spaces. J. Tits showed that there are no nontrivial Clifford translations of symmetric spaces of noncompact type; and it is easy to see that the Clifford translations of Euclidean space are just the ordinary translations.

One of the main results of the above work is that if $M$ is any simply connected symmetric space and $\Gamma$ is a properly discontinuous group of isometries of $M$, then a necessary and sufficient condition for $M / \Gamma$ to be homogeneous is that $\Gamma$ consists of Clifford translations. An equivalent statement is that $\Gamma$ consists of Clifford translations if and only if its centralizer in the isometry group of $M$ is transitive on $M$.

All the methods used so far have involved a case by case check of the symmetric spaces (of compact type) in Cartan's list, with each case requiring different techniques.

In this paper we give a direct proof of the above result for cyclic groups $\Gamma$ acting on symmetric spaces of compact type. The proof relies on a geodesic characterization of Clifford translations (Theorem 1.6), together with extensions of some arguments in [2], and makes no use of the classification. (The results in [2] give the same answer for symmetric spaces

Received by the editors July 16, 1973.

AMS (MOS) subject classifications (1970). Primary 53C35, 53C30; Secondary $53 \mathrm{C} 70$.

Key words and phrases. Clifford translation, isometry, symmetric space, homogeneous space. 
of noncompact type, but they are vacuous in view of the theorems of Tits.) The method does not seem to work for noncyclic groups.

(1) A geodesic characterization of Clifford translations. Let $M$ be a complete connected $C^{\infty}$ Riemannian manifold with Riemannian inner product $\langle\cdots, \cdots\rangle$ and distance $\rho$. If $f: M \rightarrow M$ is any function, then the displacement function $\delta_{f}: M \rightarrow \boldsymbol{R}$ of $f$ is given by $\delta_{f}(x)=\rho(x, f(x))$ for all $x \in M$.

(1.1) Definition. If $f: M \rightarrow M$ is a function and $\gamma: \boldsymbol{R} \rightarrow M$ is a $C^{\infty}$ curve parametrized proportional to arc-length, then $f$ preserves $\gamma$ if there is a number $a \in \boldsymbol{R}$ such that $f(\gamma(t))=\gamma(t+a)$ for all $t$.

The following lemma is easy to verify.

(1.2) Lemma. Let $f: M \rightarrow M$ be an isometry, $\gamma: \boldsymbol{R} \rightarrow M$ a geodesic parameterized proportional to arc-length, and $a \in \boldsymbol{R}$ a constant. The following are equivalent:

(i) $f(\gamma(t))=\gamma(t+a)$ for all $t$;

(ii) $f_{*}\left(\gamma^{\prime}(t)\right)=\gamma^{\prime}(t+a)$ for some $t$;

(iii) $f_{*}\left(\gamma^{\prime}(t)^{\perp}\right)=\gamma^{\prime}(t+a)^{\perp}$ and $\left\langle f_{*}\left(\gamma^{\prime}(t)\right), \gamma^{\prime}(t+a)\right\rangle \geqq 0$ for some $t$.

(1.3) Definition. Let $f: M \rightarrow M$ be an isometry and $x \in M$. Then

(i) $f$ satisfies $\left(P_{x}\right)$ if there is at least one minimizing geodesic $\gamma$ from $x$ to $f(x)$ which is preserved by $f$.

(ii) $\operatorname{Pres}(f)=\left\{x \mid f\right.$ satisfies $\left.\left(P_{x}\right)\right\}$.

Note. In [2] we investigated the set $\operatorname{Crit}(f)$ of critical points of the function $x \mapsto \delta_{f}^{2}(x)$ under the assumption that $f$ has small displacement (i.e. $f(x)$ is not in the cut locus of $x$ for any $x \in M)$. We showed there that $\operatorname{Crit}(f)$ is precisely the set of $x$ for which $f$ satisfies $\left(P_{x}\right)$. The small displacement condition is too strong for many situations of interest, and the more natural object geometrically is $\operatorname{Pres}(f)$. It has meaning even in the generality of the $G$-spaces of Busemann.

(1.4) Proposition. Let $f, h: M \rightarrow M$ be isometries. Then

(i) $f$ satisfies $\left(P_{h x}\right)$ if and only if $h^{-1}$ fh satisfies $\left(P_{x}\right)$;

(ii) $h x \in \operatorname{Pres}(f)$ if and only if $x \in \operatorname{Pres}\left(h^{-1} f h\right)$;

(iii) $h \cdot \operatorname{Pres}(f)=\operatorname{Pres}\left(h f h^{-1}\right)$.

ProOF. See [2].

(1.5) Definition. An isometry $f: M \rightarrow M$ is a Clifford translation if $\delta_{f}: x \mapsto \rho(x, f(x))$ is constant on $M$.

(This is called a Clifford-Wolf isometry in [1].)

(1.6) THEOREM. Let $f: M \rightarrow M$ be an isometry. The following are equivalent:

(i) $f$ is a Clifford translation; 
(ii) for each $x \in M$ there is a minimizing geodesic $\gamma$ from $x$ to $f(x)$ preserved by $f($ i.e. $\operatorname{Pres}(f)=M)$;

(iii) for each $x \in M$ and every minimizing geodesic $\gamma$ from $x$ to $f(x)$, f preserves $\gamma$.

Proof. Suppose (ii) holds, $x \in M$, and $\gamma$ is a minimizing geodesic from $x$ to $f(x)$ preserved by $f$. Assume $\gamma$ is parameterized by arc-length, and let $a=\rho(x, f(x))$. Let $b>0$, and $Q:[0, a] \times(-b, b) \rightarrow M$ a $C^{\infty}$ variation of $\gamma$ such that:

(i) the curve $t \mapsto Q(t, 0)$ is the curve $\gamma$;

(ii) $f(Q(0, s))=Q(a, s)$ for all $s$;

(iii) each curve $t \mapsto Q_{s}(t)=Q(t, s)$ is parameterized proportional to arc-length. Let $X=Q_{*} \partial / \partial s, \quad Y=Q_{*} \partial / \partial t$, and $L\left(Q_{s}\right)=\int_{0}^{a}\langle Y, Y\rangle^{1 / 2} d t$. The formula for first variation of arc-length says:

$$
\left(d /\left.d s\right|_{s=0}\right) L\left(Q_{s}\right)=\left\langle X_{\gamma(a)}, Y_{\gamma(a)}\right\rangle-\left\langle X_{\gamma(0)}, Y_{\gamma(0)}\right\rangle-\int_{0}^{a}\left\langle X, \nabla_{Y} Y\right\rangle d t .
$$

$\nabla_{Y} Y \equiv 0$ since $\gamma$ is a geodesic; and since $f$ preserves $\gamma, f_{*} Y_{\gamma(0)}=f_{*} \gamma^{\prime}(0)=$ $\gamma^{\prime}(a)=Y_{\gamma(a)}$. Therefore,

$$
\left\langle X_{\gamma(a)}, Y_{\gamma(a)}\right\rangle=\left\langle f_{*} X_{\gamma(0)}, f_{*} Y_{\gamma(0)}\right\rangle=\left\langle X_{\gamma(0)}, Y_{\gamma(0)}\right\rangle
$$

so $\left(d /\left.d s\right|_{s=0}\right) L\left(Q_{s}\right)=0$. For each $s \in(-b, b)$, we have $L\left(Q_{s}\right) \geqq \delta_{f}(Q(0, s))$ and $L\left(Q_{0}\right)=L(\gamma)=\delta_{f}(Q(0,0))$ so $L\left(Q_{s}\right)-L\left(Q_{0}\right) \geqq \delta_{f}(Q(0, s))-\delta_{f}(Q(0,0))$. Since $\lim _{s \rightarrow 0}(1 / s)\left(L\left(Q_{s}\right)-L\left(Q_{0}\right)\right)=0$ it follows that for each $\varepsilon>0$ there is $\delta>0$ such that if $0<s<\delta$ then

$$
(1 / s)\left(\delta_{f}(Q(0, s))-\delta_{f}(Q(0,0))\right) \leqq(1 / s)\left(L\left(Q_{s}\right)-L\left(Q_{0}\right)\right)<\varepsilon .
$$

By replacing $Q$ by $\widetilde{Q}(t, s)=Q(t,-s)$ we see that

$$
(1 /|s|)\left(\delta_{f}(Q(0, s))-\delta_{f}(Q(0,0))\right)<\varepsilon \text { for all } 0<|s|<\delta .
$$

Now assume the curves $s \mapsto Q(0, s), s \mapsto Q(a, s)$ are geodesics parameterized by arc-length, so that $|s|=\rho(Q(0,0), Q(0, s))$ for small $|s|$. Then for each geodesic $v$ emanating from $x$ and for each $\varepsilon>0$, there is $\delta>0$ such that if $y$ lies on $v$ and $\rho(x, y)<\delta$, then $\delta_{f}(y)-\delta_{f}(x)<\varepsilon \cdot \rho(x, y)$. Since $\delta$ depends only on $x, v$, and $\varepsilon$, we can restrict to a suitable neighborhood $C$ of $x$ and obtain $\delta>0$ which depends only on $\varepsilon$. Thus, for each $\varepsilon>0$ there is $\delta>0$ such that if $x, y \in C$ and $\rho(x, y)<\delta$, then $\delta_{f}(y)-$ $\delta_{f}(x)<\varepsilon \cdot \rho(x, y)$. By symmetry we get $\left|\delta_{f}(y)-\delta_{f}(x)\right|<\varepsilon \cdot \rho(x, y)$, so $\delta_{f}$ has zero derivative in $C$. Since $M$ is connected and covered by the neighborhoods $C, \delta_{f}$ is constant on $M$.

If $f$ is a Clifford translation, then it follows easily that $\left(d /\left.d s\right|_{s=0}\right) L\left(Q_{s}\right)=0$ for every variation $Q$ satisfying (i), (ii), (iii). Then $\left\langle X_{f(x)}, \gamma^{\prime}(a)\right\rangle=\left\langle X_{x}, \gamma^{\prime}(0)\right\rangle$ 
so if $X_{x} \perp \gamma^{\prime}(0)$ then $X_{f(x)} \perp \gamma^{\prime}(a)$. This proves that $f_{*}\left(\gamma^{\prime}(0)^{\perp}\right)=\gamma^{\prime}(a)^{\perp}$. If $X=Y$ the first variation formula implies that $0\left\langle\langle X, Y\rangle_{(0,0)}=\langle X, Y\rangle_{(a, 0)}\right.$, so (iii) of Lemma 1.2 holds. Then $f$ preserves $\gamma$, proving (iii). Since (iii) implies (ii) the proof is complete. Q.E.D.

(2) Clifford translations of symmetric spaces. Assume now that $M$ is a normal Riemannian homogeneous space, $G=I(M)$ is its full isometry group, and $K=I_{x}(M)$ is the isotropy subgroup of $G$ at $x \in M$. We identify $x$ with $K$. Let $\mathfrak{g}$, $\mathfrak{f}$ be their Lie algebras, and $\mathfrak{g}=\mathfrak{f}+\mathfrak{m}$ an $\operatorname{ad}(K)$-invariant splitting of $\mathfrak{g}$. Provide $m$ with an $\operatorname{ad}(K)$-invariant inner product corresponding to the Riemannian inner product $\langle\cdots, \cdots\rangle$ on $T_{x} M$ via the usual identification $T_{x} M \leftrightarrow \mathrm{m}$. Normality of $M$ means the geodesics emanating from $x$ have the form $t \mapsto(\exp t Y) \cdot x$ with $Y \in \mathfrak{m}$. If $M$ is a symmetric space and $s_{x} \in K$ is the geodesic symmetry at $x$ then the splitting $\mathfrak{g}=\mathfrak{f}+\mathfrak{m}$ is assumed to be into the \pm 1 eigenspaces of the involution $\sigma=\operatorname{ad}\left(s_{x}\right): \mathfrak{g} \rightarrow \mathfrak{g}$. $\sigma$ will also denote the involution $\sigma=\operatorname{ad}\left(s_{x}\right): G \rightarrow G$.

(2.1) Proposition. Let $M$ be a normal Riemannian homogeneous space and $g \in I(M)$. Then:

(i) $g$ satisfies $\left(P_{x}\right)$ if and only if $g=(\exp Y) k$, where $Y \in m$ is such that $t \mapsto(\exp t Y) \cdot x$ is a minimizing geodesic from $x$ to gx, and $k \in K$ is such that $\operatorname{ad}(k) Y=Y$.

(ii) $\operatorname{Pres}(g)=\left\{h^{-1} \cdot x \mid h g h^{-1}=\left(\exp Y_{h}\right) k_{n}\right.$ as in (i) $\}$.

Proof. See [2].

Suppose now that $M$ is a compact symmetric space, $x \in M$ is fixed, and $\sigma=\operatorname{ad}\left(s_{x}\right)$ is the involution of $G$ and $\mathfrak{g}$. Note that $\operatorname{ad}(k)=\operatorname{ad}(\sigma k)$ for all $k \in K$, although we need not have $\sigma k=k$.

Let Aut $(\mathfrak{g})$ be the Lie group of all automorphisms of $\mathfrak{g}$, and aut $(\mathfrak{g})$ its Lie algebra. The involution $\sigma$ of $\mathfrak{g}$ induces an involution $\tilde{\sigma}$ of Aut $(\mathfrak{g})$ (and aut(g)) by: $\tilde{\sigma} \mu=\sigma \circ \mu \circ \sigma$ for all $\mu \in \operatorname{Aut}(\mathfrak{g})$. The subgroup $\operatorname{ad}(G) \subset$ Aut $(\mathfrak{g})$ is $\tilde{\sigma}$-invariant and $\operatorname{ad}(K)$ is pointwise fixed by $\tilde{\sigma}$.

If $G$ is any compact Lie group (not assumed connected), it has certain abelian subgroups $S$ such that:

(i) there is $g \in S$, called a generator, whose powers are dense in $S$.

(ii) $S$ has finite index in its normalizer in $G$.

These groups are obtained by the following construction (see, for example, [3] or [4]): Take any $g \in G$, and let $S^{0}$ be a maximal torus of the centralizer $Z(g)$ of $g$ in $G$. Then the required group $S$ is the one generated by $S^{0}$ and $g$.

For $G$ connected, the groups $S$ are the maximal tori of $G$; but they need not be of maximal rank nor connected if $G$ is not connected. These groups are denoted by $T^{(h)}$ in de Siebenthal [4], and they are called Cartan subgroups by G. Segal in [3]. Current usage gives a different meaning to 
"Cartan subgroup", so we will refer to the groups $S$ above as $T^{(h)}$-subgroups.

(2.2) Lemma. Let $M$ be a compact symmetric space and $g \in I(M)$. If $x(=e K)$ lies in $\operatorname{Pres}(g)$ then there is a $T^{(h)}$-subgroup $S$ of $I(M)$ such that:

(i) $g \in S$,

(ii) $\sigma S^{0}=S$ ( $S^{0}=$ identity component of $S$ ),

(iii) $S x=S^{0} x$ (i.e. $S$ has connected $x$-orbit).

Proof. Let $g=(\exp Y) k$ be a splitting as in $(2.1)$, and let $Z(k)$ be the centralizer of $k$ in $I(M)$, and $z_{k}$ its Lie algebra. $z_{k}$ is the +1 eigenspace of $\operatorname{ad}(k)$ acting on $\mathfrak{g}$, and since $\operatorname{ad}(k)=\operatorname{ad}(\sigma k)$ it follows that $3_{k}=3_{\sigma k}=\sigma_{3 k}$ so $z_{k}$ is $\sigma$-invariant. Therefore $z_{k}$ splits into the \pm 1 eigenspaces of $\sigma$ : $3_{k}=3_{k}^{+}+\bar{k}_{\bar{k}}$. Evidently $Y \in 3_{\bar{k}}$. Let $A \subset 3_{\bar{k}}$ be a maximal abelian subspace containing $Y$, and let $B$ be a maximal abelian subspace of the centralizer of $A$ in $3_{k}^{+}$. If $X \in 3_{k}$ centralizes $A+B$ then $[X, A]=0=[X, B]$ so if we decompose $X=X^{+}+X^{-}$then $0=\left[X^{+}, A\right]+\left[X^{-}, A\right]$ and $0=\left[X^{+}, B\right]+\left[X^{-}, B\right]$. Therefore $\left[X^{-}, A\right]=0$ so $X^{-} \in A$. Since $X^{+}$centralizes both $A$ and $B$, $X^{+} \in B$ so $X \in A+B$ and $A+B$ is a maximal abelian subalgebra of $3_{k}$. $A+B$ is clearly $\sigma$-invariant.

Let $S^{0}=\exp (A+B)$, so $\sigma S^{0}=S^{0}$, and let $S$ be the $T^{(h)}$-subgroup of $G$ generated by $S^{0}$ and $k$. Then $\exp Y, k,(\exp Y) \cdot k=g \in S$. The cosets of $S^{0}$ in $S$ are represented by powers of $k$ so $S \cdot x=S^{0} \cdot x$, proving the lemma. Q.E.D.

(2.3) Proposition. Let $M$ be a compact symmetric space and $g \in I(M)$. Then there is a set $\left\{g_{\beta}\right\}$ of generators of the $T^{(h)}$-subgroups of $I(M)$ containing $g$ such that $\operatorname{Pres}(g) \subset \bigcup_{\beta} \operatorname{Pres}\left(g_{\beta}\right)$.

Proof. Suppose $x \in \operatorname{Pres}(g)$ (we may assume $x=e K$ ). By Lemma 2.2 there is a $T^{(h)}$-subgroup $S$ satisfying (i), (ii), (iii). Let $g=(\exp Y) k$ be the usual splitting of $g$. There is a finite set of powers $k^{a}$ of $k$ such that $S=\bigcup_{a} S^{0} k^{a}$. Since $S$ is the product of $S^{0}$ with a finite cyclic group generated by an element $h \in S$, and the generators of $S$ include all the products $g_{0} h$ where $g_{0}$ is a generator of $S^{0}$, it follows that one of the components of $S$ has a dense set of generators of $S$. Say that component is $S^{0} k^{a}$. Then there is a generator $g_{\beta}=p_{\beta} k^{a}$ of $S$ with $p_{\beta} \in S^{0}$ as near $e$ as we like. Now $S^{0} x$ is a totally geodesic submanifold of $M$, so we can choose $g_{\beta}$ so the unique minimizing geodesic going from $x$ to $g_{\beta} x$ lies in $S^{0} x$. Then if $Y_{\beta} \in m$ is the tangent to this geodesic at $x, Y_{\beta}$ must lie in the Lie algebra of $S^{0}$. Thus, $\left(\exp Y_{\beta}\right) x=g_{\beta} x$ so $g_{\beta}=\left(\exp Y_{\beta}\right) k_{\beta}$ for some $k_{\beta} \in K$. But $k_{\beta} \in S$, so $\operatorname{ad}\left(k_{\beta}\right) Y_{\beta}=Y_{\beta}$, proving that $x \in \operatorname{Pres}\left(g_{\beta}\right)$. This argument works for all $x \in \operatorname{Pres}(g)$ so the proof is complete. Q.E.D. 
(2.4) Corollary. If $g$ is a Clifford translation then $M=\bigcup_{\alpha} \operatorname{Pres}\left(g_{\alpha}\right)$, where $\left\{g_{\alpha}\right\}$ is a set of generators of the $T^{(h)}$-subgroups $\left\{S_{\alpha}\right\}$ containing $g$.

Now assume $M$ is symmetric of compact type.

(2.5) Proposition. If $g \in G$ generates a $T^{(h)}$-subgroup $S$, then $\operatorname{Pres}(g)=$ $\bigcup_{i=1}^{n} S^{0} \cdot x_{i}$, where $\left\{x_{i}\right\}_{i=1}^{n}$ is a set of representatives of the components of $\operatorname{Pres}(g)$.

Proof. Let $x \in \operatorname{Pres}(g)$ and $g=(\exp Y) k$ the usual splitting. If $h \in \exp m$ is such that $h^{-1} x \in \operatorname{Pres}(g)$ then the $T^{(h)}$-subgroup $h S h^{-1}$ satisfies (ii) of Lemma 2.2. Moreover, $h S h^{-1}$ is generated by $h S^{0} h^{-1}$ and the component $k \in K$ of the splitting $h g h^{-1}=\left(\exp Y_{h}\right) k_{h}$. Since $h S^{0} h^{-1}$ is $\sigma$-invariant then $\operatorname{ad}\left(h S^{0} h^{-1}\right)$ is $\tilde{\sigma}$-invariant (and $\operatorname{ad}\left(k_{h}\right)$ is $\tilde{\sigma}$-stable). Therefore $\operatorname{ad}\left(h S h^{-1}\right)$ is $\tilde{\sigma}$-invariant. $\operatorname{Then} \tilde{\sigma}\left[\operatorname{ad}\left(h S h^{-1}\right)\right]=\operatorname{ad}\left(h S h^{-1}\right)$ so

$$
\operatorname{ad}\left(h^{-1}\right) \operatorname{ad} S \operatorname{ad}(h)=\operatorname{ad}(h) \operatorname{ad} S \operatorname{ad}\left(h^{-1}\right),
$$

since $\operatorname{ad} S$ is also $\tilde{\sigma}$-invariant. Thus, $\operatorname{ad}\left(h^{2}\right) \operatorname{ad} S \operatorname{ad}\left(h^{2}\right)^{-1}=\operatorname{ad} S$ and $\operatorname{ad}\left(h^{2}\right)$ is in the normalizer of ad $S$ in ad $G$. Since the kernel of ad: $G \rightarrow$ Aut $(\mathrm{g})$ is finite (being the centralizer of $G^{0}$ in $G$ ), it follows that for $h$ sufficiently near $e$, the above condition implies that $h^{2} S\left(h^{2}\right)^{-1}=S$. For $h$ near $e$, this means $h \in S^{0}$ since $S$ has finite index in its normalizer and the only elements of $S$ near $e$ lie in $S^{0}$. Therefore, for all $h \in \exp \mathrm{m}$ sufficiently near $e, h^{-1} x \in \operatorname{Pres}(g)$ if and only if $h \in S^{0}$. This argument is independent of the point $x \in \operatorname{Pres}(g)$ originally chosen, so $S^{0} \cdot x$ is open in $\operatorname{Pres}(g)$. Since $S^{0} \cdot x$ is also closed in $\operatorname{Pres}(g)$ it is a component. The number of components is finite since $\operatorname{Pres}(g)$ is an imbedded submanifold of a compact manifold. Q.E.D.

(2.6) Proposition. For each $g \in G$, Pres $(g)=\bigcup_{i=1}^{n} Z^{0}(g) \cdot x_{i}$, where $\left\{x_{i}\right\}_{i=1}^{n}$ is a set of representatives of the components of $\operatorname{Pres}(g)$, and $Z^{0}(g)$ is the identity component of the centralizer of $g$ in $G$.

Proof. Let $\left\{S_{\alpha}\right\}$ be the $T^{(h)}$-subgroups of $G$ containing $g$. Their identity components $S_{\alpha}^{0}$ are the maximal tori of the compact Lie group $Z^{0}(g)$, so they are all conjugate within $Z^{0}(g)$. Thus there are elements $h_{\alpha} \in Z^{0}(g)$ such that $\left\{h_{\alpha} S_{\alpha_{0}}^{0} h^{-1}\right\}$ is the set of all maximal tori in $Z^{0}(g)$, where $S_{\alpha_{0}}^{0}$ is some fixed one of them. Then $\left\{h_{\alpha} S_{\alpha_{0}} h_{\alpha}^{-1}\right\}=\left\{S_{\alpha}\right\}$ is the set of all $T^{(h)}$-subgroups containing $g$. Let $\left\{x_{i}\right\}_{i=1}^{n}$ be a set of representatives of the components of $\operatorname{Pres}\left(g_{\alpha_{0}}\right)$, where $g_{\alpha_{0}}$ is a generator of the $T^{(h)}$-subgroup $S_{\alpha_{0}}$. Then

$$
\operatorname{Pres}\left(g_{\alpha}\right)=\operatorname{Pres}\left(h_{\alpha} g_{\alpha_{0}} h_{\alpha}^{-1}\right)=h_{\alpha} \operatorname{Pres}\left(g_{\alpha_{0}}\right)=\bigcup_{i=1}^{n} h_{\alpha} S^{0} \cdot x_{i} ;
$$


so

$$
\begin{aligned}
\operatorname{Pres}(g) & \subset \bigcup_{\alpha} \operatorname{Pres}\left(g_{\alpha}\right) \\
& =\bigcup_{\alpha} \bigcup_{i=1}^{n} h_{\alpha} S^{0} \cdot x_{i}=\bigcup_{i=1}^{n}\left(\bigcup_{\alpha} h_{\alpha} S^{0}\right) \cdot x_{i} \subset \bigcup_{i=1}^{n} Z^{0}(g) \cdot x_{i} .
\end{aligned}
$$

The other inclusion is obvious. Q.E.D.

(2.7) Corollary. $g \in I(M)$ is a Clifford translation if and only if $Z^{0}(g)$ is transitive on $M$.

Proof. If $Z^{0}(g)$ is transitive then for each $y \in M, y=u x$ for some $u \in Z^{0}(g)$. Then $\rho(y, g y)=\rho(u x, g u x)=\rho(u x, u g x)=\rho(x, g x)$ so $g$ has constant displacement. Conversely, if $g$ is a Clifford translation then $M=\bigcup_{i=1}^{n} Z^{0}(g) \cdot x_{i}$ is a finite union of closed subsets, so by the Baire category theorem one of them is open. Thus $Z^{0}(g)$ has an open orbit which is all of $M$ since $M$ is connected. Q.E.D.

(2.8) CoRollary. A generator of a $T^{(h)}$-subgroup cannot be a Clifford translation of a symmetric space of compact type.

PRoof. If $g$ generates a $T^{(h)}$-subgroup and is a Clifford translation then $Z^{0}(g)$ is a $\sigma$-invariant transitive torus, implying that $M$ is flat. Q.E.D.

(2.9) COROLlary. If $\Gamma$ is a cyclic group of Clifford translations acting properly discontinuously on a symmetric space of compact type then $M / \Gamma$ is homogeneous.

REMARK. The converse has a simple proof found for example in [8].

\section{REFERENCES}

1. H. Freudenthal, Clifford-Wolf-Isometrien symmetrischer Räume, Math. Ann. 150 (1963), 136-149. MR 27 \#693.

2. V. Ozols, Critical points of the displacement function of an isometry, J. Differential Geometry 3 (1969), 411-432. MR 42 \#1010.

3. G. Segal, The representation ring of a compact Lie group, Inst. Hautes Études Sci. Publ. Math. 34 (1968), 113-128. MR 42 \#1529.

4. J. de Siebenthal, Sur les groupes de Lie compacts non connexes, Comment. Math. Helv. 31 (1956), 41-89. MR 20 \#926.

5. G. Vincent, Les groupes linéaires finis sans points fixes, Comment. Math. Helv. 20 (1947), 117-171. MR 9, 131.

6. J. A. Wolf, Vincent's conjecture on Clifford translations of the sphere, Comment. Math. Helv. 36 (1961), 33-41. MR 25 \#532.

7. - Locally symmetric homogeneous spaces, Comment. Math. Helv. 37 (1962/63), 65-101. MR 26 \#5522.

8. - Spaces of constant curvature, 2nd ed., 1972.

Department of Mathematics, University of Washington, Seattle, Washington 98195 